\title{
Comparison of two epidemiological protocols for measuring airway responsiveness and allergic sensitivity in adults
}

\author{
B.G. Toelle*, J.K. Peat*, C.M. Salome*, J. Crane**, D. McMillan**, \\ J. Dermand+, W. D'Souza**, A.J. Woolcock*
}

Comparison of two epidemiological protocols for measuring airway responsiveness and allergic sensitivity in adults. B.G. Toelle, J.K. Peat, C.M. Salome, J. Crane, D. McMillan, J. Dermand, W. D'Souza, A.J. Woolcock. (CERS Journals Ltd 1994.

ABSTRACT: In recent years, airway responsiveness has commonly been measured in epidemiological studies using one of two methods. In one method, histamine is administered via a handheld DeVilbiss nebulizer and in the other, methacholine is administered via a dosimeter. Allergic sensitivity has commonly been measured by either the allergen droplet method or by Phazet. We wanted to assess the comparability of airway responsiveness and of allergic sensitivity measured by both methods.

A total of 48 volunteers, including normal and asthmatic subjects, participated in the study. Subjects first underwent one of the two tests of airway responsiveness and allergic sensitivity, and then returned within $\mathbf{1 0}$ days to undergo tests using the second protocol. Commencement protocol was allocated in random order.

There was good agreement between both methods for assessing airway responsiveness and for assessing allergic sensitivity. The difference for dose response ratio (DRR) between histamine and methacholine was a 1.19 (95\% (CI) $0.78,1.82)$ fold changes, which was not statistically significant. Agreement between allergic sensitivity methods was perfect for Alternaria tenuis, good for rye-grass $(k a p p a=0.71)$ and moderate for cat and Dermatophagoides pteronyssinus (kappa approximately 0.5). It is possible to compare data from epidemiological studies which use these methods.

Eur Respir J., 1994, 7, 1798-1804.
*Dept of Medicine, University of Sydney, Sydney, NSW, Australia. **Dept of Medicine, Wellington School of Medicine, Wellington, New Zealand. +Royal Prince Alfred Hospital, Camperdown, NSW, Australia.

Correspondence: AJ. Woolcock Institute of Respiratory Medicine Royal Prince Alfred Hospital

Camperdown

NSW 2050

Australia

Keywords: Allergy

asthma

bronchial hyperreactivity

bronchial provocation tests epidemiology

Received: November 161993

Accepted after revision June 181994
Although a great deal of effort has been given to standardizing questionnaires used in the assessment of asthma in communities [1], a wide variety of objective methods are currently used to measure airway responsiveness and allergic sensitivity. Epidemiological protocols vary in their use of methods for measuring airway responsiveness and allergic sensitivity.

Many studies, in several countries, have used a protocol which measures airway responsiveness using histamine with the rapid method [2-5]. In adults, histamine and methacholine appear to be equipotent in field studies [6], and in asthmatics tested in the clinic [7], but not in patients with chronic obstructive lung disease [8]. However, a population study of children showed that asthmatics were slightly more sensitive to histamine than to methacholine [9], so that results using different agents may not be directly comparable. In addition to measuring airway responsiveness, allergic sensitivity has commonly been measured using the method of PEPYs [10], in which droplets of selected allergens are applied to the forearm and the skin pricked through the drop with a lancet.

A major screening of populations, the European Commission (EC) Respiratory Health Survey [11] has used a protocol which measures airway responsiveness using methacholine administered by dosimeter. This method has the advantage that higher doses can be given with fewer side-effects [12]. In this protocol, allergic sensitivity has been measured using allergen coated lancets (Phazet) [13], which are thought to more accurately standardize the dose of allergen that is delivered under the skin.

In order to determine the comparability of the most widely used epidemiological protocols to measure both airway responsiveness and allergic sensitivity in epidemiological studies, we conducted a study of adults using each of two methods for each measurement within a 10 day interval. In this paper, we report results from the two different methods for assessing airway responsiveness (histamine by rapid method versus methacholine by dosimeter) and allergic sensitivity (allergen droplet versus Phazet).

\section{Methods}

\section{Population}

In September 1991, and in March 1992, both asthmatic and nonasthmatic research volunteers in Wellington, 
New Zealand were invited to join a study to compare two different protocols for measuring asthma and allergy. Subjects were contacted by telephone, at which time the study design was explained and an appointment for testing arranged. Because the data were paired, it was calculated that a minimum of 33 subjects were required to detect a significant difference in dose-response ratio (DRR) between protocols at the $\mathrm{p}<0.05$ level (power $80 \%$ ), or 44 subjects at the $\mathrm{p}<0.01$ level. A total of 48 subjects consented to be involved in the study. Subjects first underwent one of the two tests of airway responsiveness (histamine delivered by the rapid method or methacholine delivered by dosimeter) and allergic sensitivity (skin-prick test to either allergen droplets or Phazets), then returned within 10 days to have similar tests using the second protocol. Commencement protocol was allocated in random order. These studies were approved by the Wellington Area Health Board Ethics Committee.

\section{Airway responsiveness}

Histamine airway challenge. Lung function was measured with the subject standing using a Mijnhardt dry rolling seal spirometer connected to a lap-top computer running Scientific and Medical software (S\&M Instrument Co. Inc., Doylestown, PA, USA) for immediate data acquisition. Subjects were asked to repeat forced expiratory manoeuvres until two readings of forced expiratory volume in one second $\left(\mathrm{FEV}_{1}\right)$ within $100 \mathrm{ml}$ of each other were obtained, of which the larger value was used in analyses. Measurements were corrected to body temperature and pressure, saturated with water vapour BTPS). Airway responsiveness was then assessed using the rapid method [14] with histamine diphosphate, which was administered by means of a DeVilbiss No. 45 handheld nebulizer in cumulative doses ranging $0.03-$ $3.9 \mu \mathrm{mol}$. Subjects were asked not to smoke in the hour before testing and to avoid taking asthma medications for $6 \mathrm{~h}$ before testing. The challenge was stopped if there was a fall in $\mathrm{FEV}_{1}$ of $20 \%$ or more from the saline value, or when the highest dose had been administered. Salbutamol aerosol $(200 \mu \mathrm{g})$ was administered to aid recovery when necessary.

Methacholine airway challenge. Lung function was measured by means of a Vitalograph compact pneumotach (Vitalograph, Bucks, UK), with the subject sitting and wearing a noseclip. Forced expiratory manoeuvres were repeated five times and the highest technically satisfactory $\mathrm{FEV}_{1}$ was recorded. Measurements were corrected to BTPS. Methacholine was administered using a dosimeter (MEFAR, Bovezzo, Italy). The challenge was carried out with cumulative doses of methacholine from 0.01 to $20.4 \mu \mathrm{mol}$. Subjects were asked not to smoke in the hour before testing and to avoid taking asthma medications for $6 \mathrm{~h}$ before testing. The challenge was stopped if the $\mathrm{FEV}_{1}$ fell by $20 \%$ or more from saline value, or when the highest dose had been admini- stered. Salbutamol aerosol $(200 \mu \mathrm{g})$ was administered to aid recovery when necessary.

Expression of results. Airway responsiveness can be assessed in populations either by the prevalence of airway hyperresponsiveness (AHR) or by the distribution of a continuous measure of responsiveness. For both challenges, a dose-response curve was obtained by plotting the percentage change in $\mathrm{FEV}_{1}$ from the saline value against the logarithm of the dose of agonist. From the curve, the provocative dose of histamine or methacholine that caused a $20 \%$ fall in $\mathrm{FEV}_{1}\left(\mathrm{PD}_{20} \mathrm{FEV}_{1}\right)$ was read. Subjects with a $\mathrm{PD}_{20} \mathrm{FEV}_{1}$ were classified as having AHR. In this comparison of results, AHR was defined firstly as a fall in FEV of $20 \%$ or more at any dose of the agonist, which is the definition used in the EC Respiratory Survey protocol, which uses methacholine. Secondly, as a fall in $\mathrm{FEV}_{1}$ of $20 \%$ or more at a dose of $3.9 \mu \mathrm{mol}$ or less, which is the definition commonly used in the histamine challenge protocol.

DRR was calculated for all subjects as the percentage fall in $\mathrm{FEV}_{1}$ at last dose divided by the total dose administered [15]. One subject who had an $\mathrm{FEV}_{1}$ of less than $60 \%$ predicted when presenting for the histamine challenge was given a bronchodilator test instead. This subject had an improvement in $\mathrm{FEV}_{1}$ following bronchodilator of $37.8 \%$, and was assigned a $\mathrm{PD}_{20} \mathrm{FEV}_{1}$ value of $0.1 \mu \mathrm{mol}$ and a DRR of $200 \%$ fall $\mathrm{FEV}_{1} / \mu \mathrm{mol}$ for inclusion in analyses.

\section{Allergic sensitivity}

Allergen droplet method. Sensitivity to eight common allergens was measured by skin-prick test reactions on the forearm using a lancet [10]. The allergens tested were produced by Hollister-Stier (Elkhart, IN). The allergens tested and the allergy units (AU) or weight/ volume $(\mathrm{W} / \mathrm{V})$ as indicated by the manufacturer were: house-dust $(1: 10 \mathrm{~W} / \mathrm{V})$; two house-dust mites Dermatophagoides pteronyssinus $\left(30,000 \mathrm{AU} \cdot \mathrm{ml}^{-1}\right)$ and $D$. farinae $(1: 50 \mathrm{~W} / \mathrm{V})$; cat dander $(1: 10 \mathrm{~W} / \mathrm{V})$; rye-grass $(1: 20 \mathrm{~W} / \mathrm{V})$; plantain $(1: 20 \mathrm{~W} / \mathrm{V})$; Alternaria tenuis $(1: 10$ $\mathrm{W} / \mathrm{V})$; and cockroach $(1: 10 \mathrm{~W} / \mathrm{V})$. Histamine and glycerol were used as positive and negative controls. If histamine tested negative or glycerol positive, the test was repeated and, if the same result occurred, the data were excluded. Allergens and control substances were stored in a refrigerator when not in use.

Phazet method. Sensitivity to 11 common allergens was measured by skin reactions on the forearm using Phazets (Pharmacia Diagnostics AB, Sweden). Phazets are lancets, precoated with allergen solution standardized for total allergenic activity $\left(100,000\right.$ biological units $\left.\cdot \mathrm{ml}^{-1}\right)$ or with histamine dihydrochloride $\left(10 \mathrm{mg} \cdot \mathrm{ml}^{-1}\right)$. The allergens tested were: cat; two moulds (Cladosporium herbarum, Alternaria tenuis); five pollens (timothy grass, birch, ragweed, parietaria, rye-grass); two dust mites (Dermatophagoides pteronyssinus, D. farinae); and olive. Histamine and an uncoated Phazet were used as the positive 
and negative controls, respectively. The Phazet was applied to the forearm at a 90 degree angle, and held in place for at least one second. Phazets were stored in a refrigerator when not in use.

Expression of results. In order to compare the allergic sensitivity methods, analysis of skin test reactions was confined to the five allergens that were common to both protocols. For both methods, wheal sizes were recorded after $15 \mathrm{~min}$ as the long axis and its perpendicular. Mean wheal size for each allergen was used in analyses. A wheal size of $4 \mathrm{~mm}$ or greater was regarded as positive [16].

Statistical methods. Data were analysed using the statistical package SAS (SAS Institute Inc., Cary, NC, USA). Both DRR and $\mathrm{PD}_{20} \mathrm{FEV}_{1}$ values are log-normally distributed [17]; therefore, they were converted to base 10 logarithms prior to analyses and the antilogarithm (geometric) means are reported. For all analyses, p-values less than 0.05 were regarded as significant and values greater than this as nonsignificant. Percentages and mean values are reported with $95 \%$ confidence intervals $(95 \%$ CI). Comparability measures between methods of measuring airway responsiveness were calculated following the method of Bland and Altman [18]. Agreement between the categorical variables, of skin-prick testing and AHR were assessed using kappa values and average correct classification rate (ACCR) [19].

\section{Results}

A total of 48 subjects were enrolled in this study. The sample consisted of 25 females and 23 males, and the mean age was 36 yrs (range 18-52 yrs). A total of $69 \%$ of the sample had ever been diagnosed as having asthma and $62 \%$ reported having had nasal symptoms such as hay fever in the last 12 months.

\section{Airway hyperresponsiveness}

Technically satisfactory data of airway responsiveness on both tests were collected from 38 subjects. Of the 10 subjects who were excluded from analysis, three subjects had used a bronchodilator within $6 \mathrm{~h}$ prior to challenge, five subjects stopped either one of the challenges before completion, one subject was unsuitable for challenge because of poor resting lung function, and one subject had an $\mathrm{FEV}_{1}$ which improved after challenge. A total of 43 subjects had technically satisfactory data of allergic sensitivity, because two subjects refused the skin test component and three subjects were excluded since they reacted to all allergens and to the negative control. Of the three subjects who reacted to all allergens, two did so only for the Phazet method and the other only for the allergen droplet method. The mean wheal size was greater than or equal to the control wheal for the Phazet method, and the majority of the wheals in the allergen droplet method were greater than the control wheal.
Baseline measurement of $\mathrm{FEV}_{1}$ was compared between the two methods. The mean difference was 0.35 $l(95 \%$ CI $0.29,0.41)$. The Vitalograph compact pneumotach spirometer measured $\mathrm{FEV}_{1}$ slightly higher than the Mijnhardt dry rolling seal spirometer. A paired $t$-test showed this difference was significant $p<0.001$. Measurements of AHR using histamine challenge by the rapid method and methacholine airway challenge by dosimeter were compared. A total of 15 subjects did not respond to either challenge, 19 responded to both challenges, and 4 responded to methacholine but not to histamine. Of the four subjects who responded to methacholine and not to histamine, two responded at the higher dose of methacholine, and the other two had a fall in $\mathrm{FEV}_{1}$ to histamine which was less than $20 \%$ and was, therefore, not sufficient to be categorized as having AHR. The mean $\mathrm{PD}_{20} \mathrm{FEV}_{1}$ for methacholine was $0.35 \mu \mathrm{mol}(95 \%$ CI $0.17,0.73)$ and the mean $\mathrm{PD}_{20} \mathrm{FEV}_{1}$, for histamine was $0.55 \mu \mathrm{mol}(95 \%$ CI $0.33,0.92)$. The proportion in agreement, average correct classification rate and kappa values for AHR are shown in table 1. A kappa value above 0.4 indicates moderate agreement and above 0.6 indicates good agreement [20]. The value for $\mathrm{PD}_{20} \mathrm{FEV}_{1}$ was calculated only for the 19 subjects who had a $20 \%$ fall in $\mathrm{FEV}_{1}$ during both challenges. The difference for histamine compared to methacholine for $\mathrm{PD}_{20} \mathrm{FEV}_{1}$ was a $0.39(95 \%$ CI $0.27,0.56)$ fold decrease. Because lower $\mathrm{PD}_{20} \mathrm{FEV}_{1}$ values indicate greater severity, these values show that subjects who had a $20 \%$ fall in $\mathrm{FEV}_{1}$ were more responsive to methacholine.

When the cut-off point defining AHR was standardized at $3.9 \mu \mathrm{mol}$, two subjects who responded to methacholine and not histamine were reclassified as nonresponders. The mean $\mathrm{PD}_{20} \mathrm{FEV}_{1}$ for methacholine by dosimeter was $0.26 \mu \mathrm{mol}(95 \%$ CI $0.14,0.47)$ and the mean $\mathrm{PD}_{20} \mathrm{FEV}_{1}$ for histamine by the rapid method was $0.55 \mu \mathrm{mol}(95 \%$ CI $0.33,0.92)$. The proportion in agreement, average correct classification rate and kappa values for AHR are shown in table 1. Thus, there was very good agreement between the two challenge methods for measuring AHR.

To compare responsiveness as a continuous variable, DRR values were calculated for all subjects irrespective of fall in $\mathrm{FEV}_{1}$. Higher DRR values indicate greater responsiveness. The mean DRR for methacholine was $6.92 \%$ fall $\mathrm{FEV}_{1} / \mu \mathrm{mol}(95 \% \mathrm{CI} 2.69,17.79)$ and mean DRR for histamine was $5.75 \%$ fall $\mathrm{FEV}_{1} / \mu \mathrm{mol}(95 \%$ CI 2.93, 11.29). The difference for histamine compared

Table 1. - Comparability of airway hyperresponsiveness in 38 subjects, for histamine and methacholine challenge

\begin{tabular}{cccc}
\hline & $\begin{array}{c}\text { Proportion } \\
\text { in agreement }\end{array}$ & $\begin{array}{c}\text { Average correct } \\
\text { classification rate }\end{array}$ & Kappa \\
\hline Final dose cut-off & 0.89 & 0.94 & 0.79 \\
& $(0.80-0.99)$ & $(0.89-1.00)$ & $(0.60-0.98)$ \\
$<3.92 \mu \mathrm{mol}$ cut-off & 0.95 & 0.97 & 0.89 \\
& $(0.88-1.00)$ & $(0.94-1.00)$ & $(0.75-1.00)$ \\
\hline
\end{tabular}

(95\% confidence intervals shown in parenthesis) 
to methacholine for DRR was a $1.19(95 \%$ CI $0.78,1.82)$ fold increases (NS). Because the confidence intervals overlap, this indicates no significant difference between results from the two challenge methods. Figure 1 shows the results from the histamine and methacholine challenge, plotted with the line of identity. In subjects with a DRR to methacholine greater than 10 , the severity of the response to methacholine was greater than to histamine, but in subjects with a DRR to methacholine less than 10 , the severity of the response to methacholine was less than to histamine. The difference in DRR value was a $2.82(95 \%$ CI $1.87,4.25)$ fold increase for the $21 \mathrm{sub}-$ jects with a DRR greater than 10 , and a 0.41 (95\% CI $0.27,0.63$ ) fold decrease for the 17 subjects with a DRR less than 10.

\section{Allergic sensitivity}

Table 2 shows the mean wheal size, mean difference and number of positive test results for each allergen by the two methods of determining allergic sensitivity. The mean wheal size was larger to the Phazet method than the allergen droplet method for histamine and all allergens except $D$. farinae. The mean difference in wheal size between methods was significantly greater for histamine by Phazet method $(\mathrm{p}<0.001)$, and for ryegrass $(\mathrm{p}<0.05)$ and $D$. farinae $(\mathrm{p}<0.001)$ by the allergen droplet method. The number of subjects that produced a wheal and the number of wheals that were categorized as positive were different between methods. The allergen droplet method produced a greater number of positive wheals for each allergen except cat dander. Also, cat dander was the only allergen to which in three

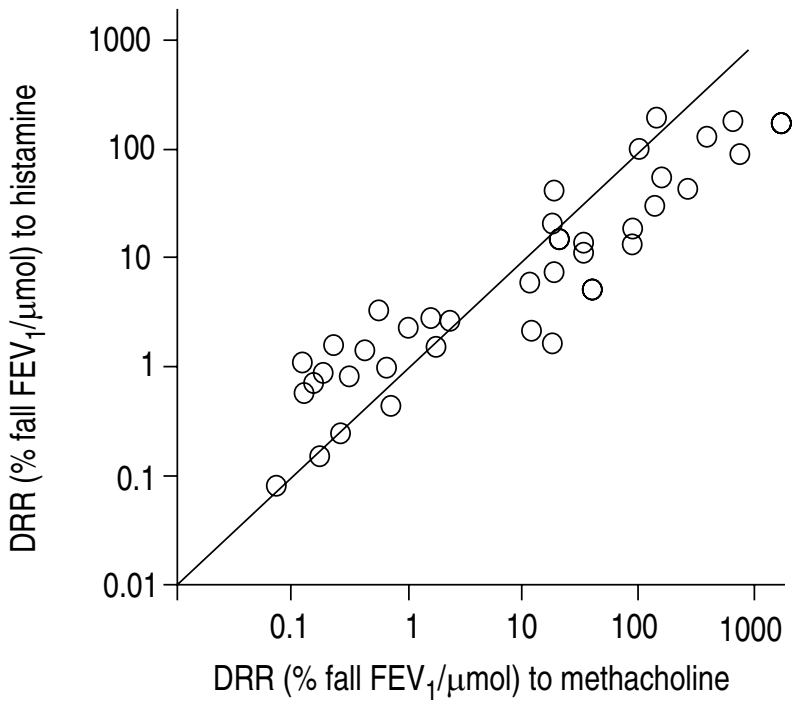

Fig. 1. - Dose response ratio (DRR) to histamine and metacholine airway challenge shown with line of identity. $\mathrm{FEV}_{1}$ : forced expiratory volume in one second.

subjects the Phazet method produced a positive wheal and the allergen droplet did not.

The comparability of the two tests of allergic sensitivity is shown in table 3 for the five allergens tested by both methods. Alternaria tenuis had perfect agreement and rye-grass had good agreement. Cat and D. pteronyssinus had moderate agreement, as indicated by kappa values of about 0.5 . The $D$. farinae allergens had poor agreement, with only $60 \%$ of subjects showing agreement for a positive test and an extremely low kappa value. Only one third of the subjects who had a positive wheal to the allergen droplet method also did to the

Table 2. - Wheal size and number of positive results for each method of assessing allergic sensitivity

\begin{tabular}{|c|c|c|c|c|c|c|}
\hline & \multicolumn{2}{|c|}{ Droplet method } & \multicolumn{2}{|c|}{ Phazet method } & \multirow{2}{*}{\multicolumn{2}{|c|}{$\begin{array}{c}\text { Mean difference } \\
(95 \% \mathrm{CI}) \\
\mathrm{mm}\end{array}$}} \\
\hline & $\begin{array}{c}\text { Mean wheal } \\
\text { size (SD) } \\
\mathrm{mm}^{\#}\end{array}$ & $\begin{array}{l}\text { Number } \\
\text { positive }^{\# \#}\end{array}$ & $\begin{array}{c}\text { Mean wheal } \\
\text { size (SD) } \\
\mathrm{mm}^{\#}\end{array}$ & $\begin{array}{l}\text { Number } \\
\text { positive }^{\# \#}\end{array}$ & & \\
\hline $\begin{array}{l}\text { Histamine } \\
\mathrm{n}\end{array}$ & $\begin{array}{l}3.8(1.0) \\
43\end{array}$ & 43 & $\begin{array}{l}6.1 \quad(2.3) \\
43\end{array}$ & 43 & $\begin{array}{c}-2.3 * * \\
43\end{array}$ & $(-3.1,-1.6)$ \\
\hline $\begin{array}{l}\text { Alternaria tenuis } \\
\mathrm{n}\end{array}$ & $\begin{array}{c}4.0 \\
8\end{array}$ & 4 & $\begin{array}{ll}7.0 & (2.7) \\
4 & \end{array}$ & 4 & $\begin{array}{c}0.5 \\
8\end{array}$ & $(-1.5,2.5)$ \\
\hline $\begin{array}{l}\text { Ryegrass } \\
\mathrm{n}\end{array}$ & $\begin{array}{l}5.4 \quad(2.3) \\
25\end{array}$ & 19 & $\begin{array}{l}5.8 \quad(3.0) \\
18\end{array}$ & 15 & $\begin{array}{l}1.2 * \\
25\end{array}$ & $(0.1,2.3)$ \\
\hline $\begin{array}{l}\text { Cat } \\
n\end{array}$ & $\begin{array}{cc}2.6 & (0.7) \\
8 & \end{array}$ & 1 & $\begin{array}{cc}4.6 & (2.5) \\
7 & \end{array}$ & 3 & $\begin{array}{r}-1.0 \\
11\end{array}$ & $(-2.7,0.7)$ \\
\hline $\begin{array}{l}\text { D. pteronyssinus } \\
\mathrm{n}\end{array}$ & $\begin{array}{l}5.7 \quad(3.0) \\
30\end{array}$ & 24 & $\begin{array}{l}6.3(3.1) \\
25\end{array}$ & 20 & $\begin{array}{c}0.5 \\
30\end{array}$ & $(-1.0,2.0)$ \\
\hline $\begin{array}{l}\text { D. farinae } \\
\mathrm{n}\end{array}$ & $\begin{array}{l}5.1 \quad(1.9) \\
26\end{array}$ & 21 & $\begin{array}{l}4.0(1.4) \\
9\end{array}$ & 4 & $\begin{array}{l}3.7 * * \\
26\end{array}$ & $(2.6,4.8)$ \\
\hline $\begin{array}{l}\text { Allergic sensitivity to any } \\
\text { of the above allergens }\end{array}$ & & 28 & & 24 & & \\
\hline
\end{tabular}

Data for wheal size are presented as mean and SD in parenthesis. \#: mean wheal size calculated from wheals $\geq 1 \mathrm{~mm}$; \#\#: number positive only includes those wheals $\geq 4 \mathrm{~mm}$. *: $\mathrm{p}<0.05$; **: $\mathrm{p}<0.001$ calculated for subjects with a wheal to either method. 
Table 3. - Comparability of allergic sensitivity measurements in 43 subjects, for allergen droplet and Phazet methods

\begin{tabular}{lccc}
\hline & $\begin{array}{c}\text { Proportion } \\
\text { in agreement }\end{array}$ & $\begin{array}{c}\text { Average correct } \\
\text { classification } \\
\text { rate }\end{array}$ & Kappa \\
\hline Alternaria tenuis & 1.00 & 1.00 & 1.00 \\
& $(1.00,1.00)$ & $(1.00,1.00)$ & $(1.00,1.00)$ \\
Rye grass & 0.86 & 0.92 & 0.71 \\
& $(0.76,0.96)$ & $(0.86,0.99)$ & $(0.50,0.92)$ \\
Cat & 0.95 & 0.98 & 0.48 \\
& $(0.89,1.02)$ & $(0.94,1.01)$ & $(0,1.10)$ \\
D. pteronyssinus & 0.77 & 0.87 & 0.54 \\
& $(0.64,0.89)$ & $(0.78,0.95)$ & $(0.29,0.79)$ \\
D. farinae & 0.60 & 0.73 & 0.19 \\
& $(0.46,0.75)$ & $(0.57,0.89)$ & $(0,0.62)$ \\
$\begin{array}{l}\text { Allergic sensitivity } \\
\text { to any of the } \\
\text { above allergens }\end{array}$ & 0.86 & 0.92 & 0.71 \\
\hline
\end{tabular}

(95\% confidence intervals in parenthesis)

Phazet method; and, of these, only half had a Phazet wheal that was classified as positive. However, overall, there was good agreement for allergic sensitivity defined as a positive wheal to any of the five allergens tested.

The distribution of wheals to the rye-grass allergen for both the allergen droplet and Phazet method is shown in figure 2 . Both methods produced wheals to the rye-grass allergen which were similarly distributed and this is also reflected in the good agreement between methods. The distribution of wheals to the $D$. pteronyssinus allergen for both the allergen droplet and Phazet method is shown in figure 3. The distribution of wheals less than $5 \mathrm{~mm}$ was similar between methods, however, for wheals $5 \mathrm{~mm}$ or greater, the distribution was different between the allergen droplet and Phazat method.

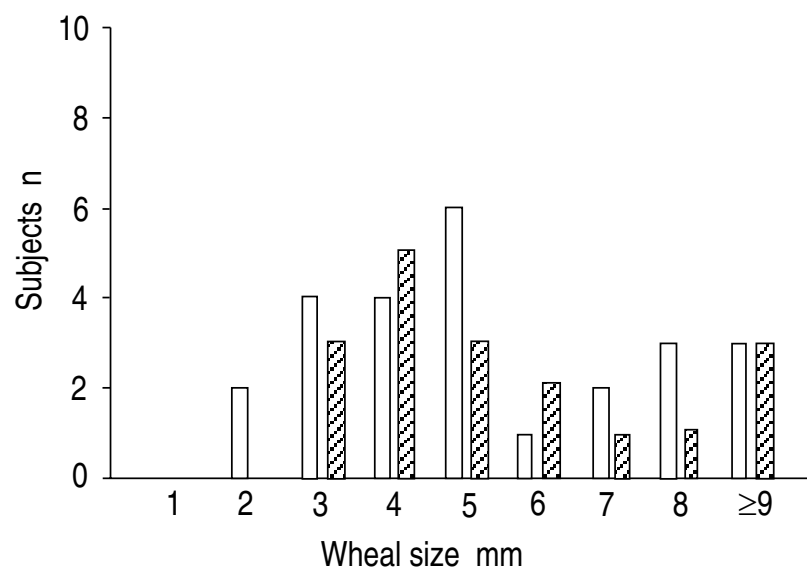

Fig. 2. - Frequency distribution of ryegrass wheals. $\bar{\square}$ : allergen droplet; $\square$ : Phazet.

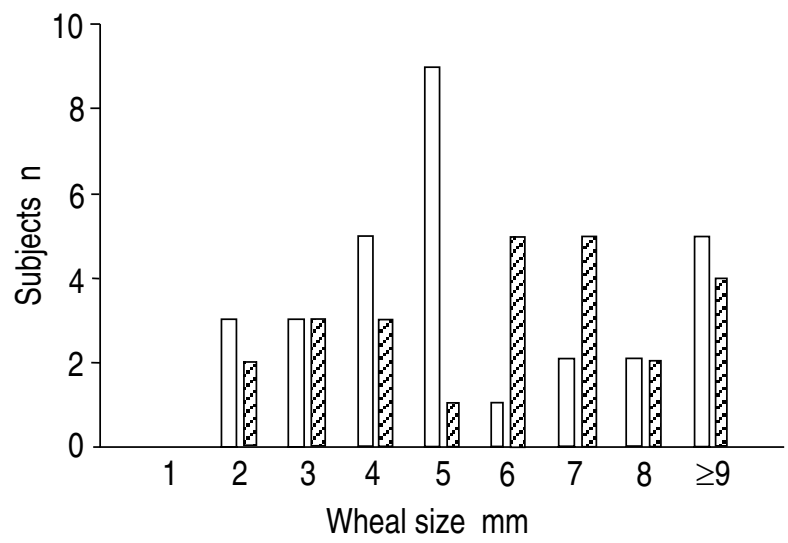

Fig. 3. - Frequency distribution of D. pteronyssinus wheals. allergen droplet; $\square$ : Phazet.

\section{Discussion}

It is important that historical data of AHR and allergic sensitivity collected in previous studies can be accurately compared with that being currently collected as part of the EC Respiratory Health Survey [11]. This study has shown that reasonable comparisons can be made between studies which have used a protocol that includes a histamine challenge using the rapid method and allergen droplet method for measuring allergic sensitivity, and the EC Respiratory Survey protocol which has used a methacholine challenge using dosimeter and Phazets for measuring allergic sensitivity. Reasonable comparisons can be made between methods of measuring airway hyperresponsiveness for calculating both DRR and $\mathrm{PD}_{20} \mathrm{FEV}_{1}$. Furthermore, when the cut-off dose determining hyperresponsiveness is standardized to $3.9 \mu \mathrm{mol}$, these methods are comparable in detecting subjects with AHR. The methods of measuring allergic sensitivity showed good overall agreement for assessing total allergic sensitivity and for comparing ryegrass, Alternaria and D. pteronyssinus allergy.

The subjects in this study included normals and asthmatics with a wide range of airway responsiveness, and atopic and nonatopic individuals. The range of DRR extended from 0.1 to $1,000 \%$ fall $\mathrm{FEV}_{1} / \mu \mathrm{mol}$ (fig. 1). The wheal sizes were reported as the geometric area which has been recommended by VOHLONEN et al. [21], and were not corrected for the size of the histamine wheal. The two airway challenge protocols have been standardized and were administered in a strict manner. Each of the protocols was administered by one of two investigators, and no investigator administered both protocols. The number of subjects was sufficient to calculate comparability with precision relative to the repeatability of the challenges.

The ability to compare results between protocols is highly dependent on the criteria used to define measurements. There has been much recent discussion about the best way to present the results of airway responsiveness, and most researchers agree that $\mathrm{PD}_{20}$ $\mathrm{FEV}_{1}$ and DRR, often called the two point slope, 
provide the most information [17, 22]. Both measures have good short-term repeatability and have good validity against symptom frequency and severity. Therefore, we have presented the results in two ways, as $\mathrm{PD}_{20} \mathrm{FEV}_{1}$ and to avoid the censoring of data, as DRR.

There was no difference in the overall comparability of the rapid method using histamine and the dosimeter method using methacholine. To ensure that the subject who was assigned a $\mathrm{PD}_{20} \mathrm{FEV}_{1}$ and $\mathrm{DRR}$ value did not distort the comparability of methods, the analysis was also performed omitting this subject's data. The overall comparability of methods was not different, with the difference in $\mathrm{PD}_{20} \mathrm{FEV}_{1}$ values changing from 0.39 to 0.36 fold decreases, and the difference in DRR values changing from 1.19 to 1.21 fold increases. There was a significant difference in $\mathrm{FEV}_{1}$ measured between protocols, but because the differences in $\mathrm{FEV}_{1}$ were linear over the entire scale this was unlikely to affect the comparability of method. Also the fall in $\mathrm{FEV}_{1}$ was standardised for baseline measurement and the order of testing was randomised to account for any change in asthmatic status. However, this suggests that lung function measurements between methods are not easily compared.

A clear difference did exist when the group with "normal" responsiveness was compared to the group with "abnormal" responsiveness [17]. The "normal" responsiveness group had a greater response to the histamine than to the methacholine protocol. This may happen because the rapid method takes only $5 \mathrm{~min}$ to administer up to the final dose, whilst the dosimeter method takes up to $15 \mathrm{~min}$ to reach this dose. BRITTON et al. [23] compared three methods of histamine challenge testing and found that the two methods which delivered the histamine in a short time had better repeatability than the longer method, suggesting that "a greater proportion of the cumulative histamine dose would be active at the end of the protocol" [23].

The "abnormal" responsiveness group had a shorter challenge time to both protocols, because they did not reach the final dose before they had a $20 \%$ fall in $\mathrm{FEV}_{1}$ and so that the greater responsiveness to methacholine may be due to the more efficient delivery of the dose. The dosimeter may deliver the dose more efficiently, but in the "normal" responsiveness group this effect may be more than offset by the cumulative effect being lost over the time taken to complete the challenge. KNOX et al. [24] compared histamine challenge by dosimeter and by rapid method, and found that in responsive subjects the responsiveness was greater to the dosimeter method than to the rapid method.

A definition of AHR using methacholine by dosimeter in the EC Respiratory Health Survey [11] has yet to be finalized, so we compared the final dose of histamine with both an equal dose and the final dose of methacholine administered to measure agreement between histamine and methacholine for classifying AHR. The agreement between methods improved slightly by using an equal dose of $3.9 \mu \mathrm{mol}$ methacholine instead of the final dose of $20.4 \mu \mathrm{mol}$. However, when comparing DRR for both methods, the final dose was used in calculations. Doses of methacholine to $20.4 \mu \mathrm{mol}$ may cause a plateau, which will alter the shape of the dose-response curve and effect the comparison with histamine [25]. There was only one subject that appeared to have a plateau to methacholine, and so the shape of all but this one curve allowed us to make comparisons of DRR at any point along the dose-response curve.

The comparability of classifying atopic individuals between methods was good overall for the five allergens common to each panel. The allergens that were found to be the most comparable are those that have the most important association with asthma [26], i.e. Alternaria tenuis, D. pteronyssinus. The agreement between methods is reassuring, considering that both the method of testing and the potency of allergen extracts used was different. The correspondence between allergy units or weight/volume and allergenic units is not known. This has led many authors to stress the importance of standardizing the dose of allergen used in tests to measure allergic sensitivity. Because more than $95 \%$ of allergic subjects are sensitized to a few common allergens, it would be helpful to standardize these allergens in panels used to define allergic sensitivity, in order to compare results using different protocols.

The mean difference in wheal size between methods was significant for histamine, rye-grass and $D$. farinae. For histamine, both methods produced the same number of wheals but, for rye-grass and $D$. farinae, the Phazet method in some subjects did not produce a wheal, and so a wheal size of 0 was used, and tended to increase the mean difference. But, when mean difference was compared only for the subjects who had a wheal to both methods the difference was significant only for histamine.

The agreement between methods for the $D$. farinae allergen was disappointing. The $D$. farinae results for the Phazet method appear to be very different from the other allergens tested by this method. The Phazet method was found to have poor comparability because it produced fewer and much smaller wheals than did the allergen droplet method. As the reactions to this allergen were very different it raises questions about the potency of this batch of Phazets for D. farinae.

The allergen droplet method consistently produced more wheals than did the Phazet method and, except for the cat allergen, produced more wheals that were classified as positive. Perhaps the greater number of wheals classified as positive can be explained by a more potent allergen extract or a more effective dose being delivered under the skin to produce a wheal. However, the exact dose of allergen delivered under the skin is not known. A recent study has found the size of a wheal to be related to the degree of trauma to the skin imposed by the method [27]. The allergen droplet method lifts up the skin as it punctures the top layer of skin, whilst the Phazet method punctures a hole directly in the skin and could be considered the less traumatic method. The smaller number of subjects with a wheal to the Phazet method could be due to the Phazet not puncturing the skin. The Phazet is pushed on the skin and held in place for a second. The difficulty is in knowing whether the Phazet has actually broken the skin, 
because in some subjects the skin is taut and in others the skin is loose. Also, in three subjects the Phazet method produced a wheal to the cat allergen, whilst the allergen droplet method did not. It is interesting that, of the multiple tests performed, this occurred only in the three subjects, and in all cases to the cat allergen.

In order to compare the prevalence of asthma and atopy worldwide, it is important that the screening protocols are standardized or at least comparable. This study has demonstrated that measurements of airway responsiveness in epidemiological studies using either of the protocols tested can be compared with confidence when the results are weighted with the comparability ratios presented. When using these protocols to compare the prevalence of AHR and allergic sensitivity, it is essential that the results are standardized for the dose administered and that a standardized panel of allergens is used.

Acknowledgements: The authors would like to thank the funding bodies in Australia (Allen \& Hanburys, the National Health and Medical Research Council of Australia, the Asthma Foundation of NSW and the Community Health and Anti-Tuberculosis Association) and in New Zealand (the New Zealand Asthma Foundation, New Zealand Lotteries Board and the Health Research Council of New Zealand) for funding this project. In addition, they thank the subjects who participated in the studies.

\section{References}

1. Burney PGJ, Chinn S, Britton JR Tattersfield AE, Papacosta AO. What symptoms predict the bronchial response to histamine? Evaluation in a community survey of the Bronchial Symptoms Questionnaire (1984) of The International Union against Tuberculosis and Lung Disease. Int J Epidemiol 1989; 18: 165-173.

2. Asher MI, Pattemore PK, Harrison AC, et al. International comparison of the prevalence of asthma symptoms and bronchial hyperresponsiveness. Am Rev Respir Dis 1988; 138: 524-529.

3. Burney PGJ, Britton JR, Chinn S, et al. Descriptive epidemiology of bronchial reactivity in an adult population: results from a community study. Thorax 1987; 42: $38-44$

4. Zhong NS, Chen RC, Yang MO, Wu ZY, Zheng JP, Li YF. Is asymptomatic bronchial hyperresponsiveness an indication of potential asthma? A two year followup of young students with bronchial hyperresponsiveness. Chest 1992; 102: 1104-1109.

5. Peat JK, Salome CM, Woolcock AJ. Factors associated with bronchial hyperresponsiveness in Australian adults and children. Eur Respir J 1992; 5: 921-929.

6. Higgins BG, Britton JR, Chinn S, et al. Comparison of histamine and methacholine for use in bronchial challenge tests in community studies. Thorax 1988; 43: 605-610.

7. Salome CM, Schoeffel RE, Woolcock AJ. Comparison of bronchial reactivity to histamine and methacholine in asthmatics. Clin Allergy 1980; 10: 541-546.

8. Du Toit J, Woolcock AJ, Salome CM, Sundrum R, Black JI. Characteristics of bronchial hyperresponsiveness in smokers with chronic airflow limitation. Am Rev Respir Dis 1986; 134: 498-501.

9. Peat JK, Salome CM, Bauman A, Toelle BG, Wachinger SL, Woolcock AJ. Repeatability of histamine bron- chial challenge and comparability with methacholine bronchial challenge in a population of Australian schoolchildren. Am Rev Respir Dis 1991; 144: 338343.

10. Pepys J. Skin tests in diagnosis. In: Gell PGH, Coombs RRA, Lachmann PJ, eds. Clinical Aspects of Immunology. 3rd edn. Oxford: Blackwell Scientific, 1975; pp. 55-80.

11. P, Burney. The European Community Respiratory Heath Survey. Eur Respir J 1994; 7: 954-960.

12. Woolcock AJ, Peat JK. Epidemiology of bronchial hyperresponsiveness. Clin Rev Allergy 1989; 7: 245256.

13. Belin L, Dreborg S, Einarsson R, et al. Phazet: ${ }^{\mathrm{TM}}$ a new type of skin-prick test. Calibration and stability. Allergy 1985; 40 (Suppl. 4): 60-63.

14. Yan K, Salome C, Woolcock AJ. Rapid method for measurement of bronchial responsiveness. Thorax 1983; 38: 760-765.

15. O'Connor G, Sparrow D, Taylor D, Segal M, Weiss S. Analysis of dose-response curves to methacholine. An approach suitable for population studies. Am Rev Respir Dis 1987; 136: 1412-1417.

16. Peat JK, Salome CM, Woolcock AJ. Longitudinal changes in atopy during a four year period: relation to bronchial hyperresponsiveness and respiratory symptoms in a population sample of Australian schoolchildren. J Allergy Clin Immunol 1990; 85: 65-74.

17. Peat JK, Salome CM, Berry G, Woolcock AJ. Relation of dose response slope to respiratory symptoms and lung function in a population study of adults living in Busselton, Western Australia. Am Rev Respir Dis 1992; 146: 860-865.

18. Bland JM, Altman DG. Statistical methods for assessing agreement between two methods of clinical rRasurement. Lancet 1986; 1: 307-310.

19. Chinn S, Burney PGJ. On measuring repeatability of data from self-administered questionnaires. Int J Epidemiol 1987; 16: 121-127.

20. Altman DG. Practical Statistics for Medical Research. 1st edn. London: Chapman and Hall, 1991; p. 404.

21. Vohlonen I, Terho EO, Koivikko A, Vanto T, Holmén A, Heinonén OP. Reproducibility of the skin-prick test. Allergy 1989; 44: 525-531.

22. Chinn S, Burney PGJ, Britton JR, Tattersfield AE, Higgins BG. Comparison of $\mathrm{PD}_{20}$ with two alternative measures of response to histamine challenge in epidemiological studies. Eur Respir J 1993; 6: 670-679.

23. Britton J, Mortagy A, Tattersfield A. Histamine challenge testing: comparison of three methods. Thorax 1986; 41: 128-132.

24. Knox AJ, Wisniewski A, Cooper S, Tattersfield AE. A comparison of the Yan and a dosimeter method for methacholine challenge in experienced and inexperienced subjects. Eur Respir J 1991; 4: 497-502.

25. Lougheed MD, Pearce-Pinto G, de Klerk NH, Ryan G, Musk AW, James A. Variability of the plateau response to methacholine in subjects without respiratory symptoms. Thorax 1993; 48: 512-517.

26. Peat JK, Woolcock AJ. Sensitivity to common allergens: relation to respiratory symptoms and bronchial hyperresponsiveness in children from three different climatic areas of Australia. Clin Exp Allergy 1991; 21: 573-581.

27. Nelson HS, Rosloniec DM, McCall LI, Iklé D. Comparative performance of five commercial prick skin test devices. J Allergy Clin Immunol 1993; 92: 750-756. 